\title{
LISTA PRELIMINAR DE LOS CURCULIONOIDEA (COLEOPTERA) DE LA COMUNIDAD DE MADRID (ESPAÑA) ${ }^{1}$
}

\author{
M. A. Alonso-Zarazaga, M. Sánchez-Ruiz y T. Domingo-Quero*
}

\begin{abstract}
RESUMEN
Se presenta por primera vez un listado de los Coleoptera Curculionoidea de la Comunidad de Madrid (España), que incluye 552 especies distribuidas en 175 géneros pertenecientes a 10 familias.

Palabras clave: Coleoptera, Curculionoidea, Madrid, lista preliminar.
\end{abstract}

\section{ABSTRACT \\ Preliminary checklist of the Curculionoidea (Coleoptera) of Comunidad de Madrid (Spain)}

A checklist of the Coleoptera Curculionoidea of Comunidad de Madrid (Spain) is presented for the first time. It includes 522 species in 175 genera belonging to 10 families.

Key words: Coleoptera, Curculionoidea, Madrid, checklist.

\section{Introducción}

Los listados de especies de Curculionoidea presentes en la Península Ibérica tienen su piedra fundacional en dos obras separadas, debido a la costumbre decimonónica de considerar por separado a los gorgojos y a los barrenillos (familias Scolytidae y Platypodidae). Durante todo el siglo XX y principios del XXI diversos autores han aportado pruebas concluyentes para considerar que los Scolytinae son el grupo hermano de la subfamilia Cossoninae de los Curculionidae y que los Platypodidae deben rebajarse a simple tribu dentro de esta última. Las obras que intentan catalogar por primera vez los Curculionoidea de la Península Ibérica son el listado de Scolytidae ibero-baleares y marroquíes de Manuel Martínez de la Escalera (1919) y la enumeración de Luis Iglesias Iglesias (1922), con muchos errores y omisiones, que completan el inacabado Catálogo de todos los Coleópteros de De la Fuente.

El listado que se presenta a continuación sólo puede considerarse como preliminar. En su elaboración se han tomado como núcleo fundamental las dos obras mencionadas anteriormente, y se ha completado esta relación con las especies mencionadas

\footnotetext{
* Depto. de Biodiversidad y Biología Evolutiva, Museo Nacional de Ciencias Naturales (CSIC), José Gutiérrez Abascal, 2; E27006 Madrid.

1 Trabajo subvencionado por el Proyecto GR/AMB/0750/2004 de la Comunidad de Madrid.
} 
en 384 obras más, cuya cita detallada sería prolija en este momento, por lo que deberá esperar a la aparición del Catálogo Ibero-balear completo, que se encuentra aún en fase de preparación. Dado que aún quedan muchas consultas por realizar y muchas obras por despojar, es obligatoria la presunción de preliminariedad.

Ante la falta de sólidas bases filogenéticas en la delimitación de los táxones de Curculionoidea, sigue esta lista la sistemática ecléctica propuesta en su momento por Alonso-Zarazaga y Lyal (1999, 2002). Aquí, sin embargo, se ha recurrido a la ordenación alfabética a ultranza, como hiciera para una lista preliminar ibero-balear Alonso-Zarazaga (2002). Los autores y fechas para los táxones de nivel familia y género pueden hallarse en los trabajos mencionados. Los endemismos ibéricos están marcados con una $\mathbf{E}$ y aquellos que sólo se conocen de la CM llevan, en añadidura, un símbolo +. Las especies seguidas de [?] corresponden a citas que no han podido ser comprobadas y que, en la mayoría de los casos, se podrá demostrar que son errores de identificación.

\section{Lista preliminar}

ANTHRIBIDAE

Anthribinae

Trigonorhinini

Trigonorhinus areolatus (Boheman, 1845)

Zygaenodini

Dissoleucas niveirostris (Fabricius, 1798)

Urodontinae

Bruchela carpetana (K. Daniel, 1906)

Bruchela pygmaea (Gyllenhal,1833)

Cercomorphus duvalii Perris, 1864

\section{APIONIDAE \\ Apioninae \\ Apionini}

Apion distincticolle Desbrochers, 1870

Apion frumentarium (Linnaeus, 1758)

Apion haematodes Kirby, 1808

Apion rubens Stephens, 1839

Apion rubiginosum Grill,1893

\section{Aplemonini}

Cistapion cyanescens (Gyllenhal,1833)

Perapion (Perapion) ilvense (Wagner,1905)

Perapion (Perapion) marchicum (Herbst, 1797)
Perapion (Perapion) violaceum violaceum (Kirby,1808)

Phrissotrichum (Phrissotrichum) joannium Ehret, 1997

Phrissotrichum (Phrissotrichum) tubiferum (Gyllenhal, 1833)

Phrissotrichum (Phrissotrichum) wenckeri (Ch. Brisout, 1863)

Pseudaplemonus chevrolati (Gyllenhal, 1833)

Pseudaplemonus limonii limonii (Kirby, 1808)

Aspidapiini

Aspidapion (Aspidapion) radiolus radiolus (Marsham, 1802)

Aspidapion (Koestlinia) aeneum (Fabricius, 1775)

Ceratapiini

Ceratapion (Acanephodus) onopordi onopordi (Kirby, 1808)

Ceratapion (Acanephodus) parens (Desbrochers, 1870)

Ceratapion (Ceratapion) armatum (Gestaecker, 1854) [?]

Ceratapion (Ceratapion) carduorum (Kirby, 1808)

Ceratapion (Ceratapion) gibbirostre (Gyllenhal, 1813)

Ceratapion (Ceratapion) damryi (Desbrochers, 1894)

Ceratapion (Clementiellus) robusticorne (Desbrochers, 1866)

Ceratapion (Echinostroma) basicorne (Illiger, 1807)

Ceratapion (Echinostroma) penetrans wanati AlonsoZarazaga, 1992

Ceratapion (Echinostroma) scalptum scalptum (Gerstäcker, 1854)

Diplapion confluens (Kirby,1808)

Exapiini

Exapion (Exapion) compactum compactum (Desbrochers, 1888)

Exapion (Exapion) difficile (Herbst, 1797) [?]

Exapion (Exapion) elongatissimum (Desbrochers, 1870) E

Exapion (Exapion) fasciolatum (Wagner, 1912) [?]

Exapion (Exapion) fuscirostre fuscirostre (Fabricius, 1775)

E+ Exapion (Exapion) laufferi laufferi (Schilsky, 1906) $\quad$ E

Exapion (Exapion) putonii (Ch. Brisout, 1866)
Lepidapion (Hidryocneme) cretaceum (Rosenhauer, 1856)

Lepidapion (Lepidapion) acuminatum (Schilsky, 1906)

Lepidapion (Lepidapion) argentatum (Gerstaecker, 1854)

Lepidapion (Lepidapion) gallaecianum (Desbrochers, 1894)

Lepidapion (Lepidapion) squamidorsum (Desbrochers, 1908)

E Lepidapion (Lepidapion) squamigerum (Jacquelin du Val, 1855) [?]

\section{Kalcapiini}

Squamapion atomarium (Kirby, 1808)

Squamapion flavimanum (Gyllenhal, 1833)

Squamapion leucophaeatum (Wencker, 1864)

Squamapion minutissimum (Rosenhauer, 1856)

Taeniapion rufulum (Wencker, 1864)

Taeniapion urticarium (Herbst,1784) 
Malvapiini

Malvapion malvae (Fabricius, 1775)

Pseudapion rufirostre (Fabricius, 1775)

Rhopalapion longirostre (Olivier, 1807)

Oxystomatini

Catapiina

Catapion pubescens (Kirby, 1811)

Catapion seniculus (Kirby, 1808)

Catapion seriatosetosulum (Wencker, 1864)

\section{Oxystomatina}

Cyanapion (Bothryorrhynchapion) afer (Gyllenhal, 1833)

Cyanapion (Cyanapion) alcyoneum (Germar, 1817)

Cyanapion (Cyanapion) columbinum (Germar, 1817)

Eutrichapion (Cnemapion) vorax (Herbst, 1797)

Eutrichapion (Eutrichapion) mystriophorum AlonsoZarazaga, 1994

Eutrichapion (Eutrichapion) viciae (Paykull, 1800) [?]

Eutrichapion (Psilocalymma) facetum (Gyllenhal, 1839) [?]

Hemitrichapion (Dimesomyops) pavidum (Germar, 1817)

Holotrichapion (Apiops) pisi (Fabricius, 1801)

Holotrichapion (Holotrichapion) ononis ononis (Kirby, 1808)

Holotrichapion (Legaricapion) aethiops (Herbst, 1797)

Holotrichapion (Legaricapion) gracilicolle (Gyllenhal, 1839)

Oxystoma craccae (Linnaeus, 1767)

Oxystoma dimidiatum (Desbrochers, 1897)

Oxystoma pomonae (Fabricius, 1798)

Oxystoma subulatum (Kirby, 1808)

Pirapion immune (Kirby, 1808)

\section{Synapiina}

Ischnopterapion (Chlorapion) virens (Herbst, 1797) Ischnopterapion (Ischnopterapion) loti (Kirby, 1808) Ischnopterapion (Ischnopterapion) cf. modestum (Germar, 1817)

Protopirapion atratulum (Germar, 1817)

Protopirapion kraatzii (Wencker, 1859)

Stenopterapion (Cobosiotherium) cantabricum (Desbrochers, 1870)

Stenopterapion (Stenopterapion) tenue (Kirby, 1808)

\section{Piezotrachelini}

Protapion assimile (Kirby, 1808)

Protapion dissimile (Germar, 1817)

Protapion interjectum interjectum (Desbrochers, 1895)

Protapion laevicolle (Kirby,1811)

Protapion nigritarse (Kirby, 1808)

Protapion schoenherri (Boheman, 1839)

Protapion trifolii (Linnaeus, 1768)

Protapion varipes (Germar, 1817)
AtTElABIDAE

Attelabinae

Attelabini

Attelabina

Attelabus nitens (Scopoli, 1763)

\section{BRACHYCERIDAE \\ Brachycerinae \\ Brachycerini}

Brachycerus barbarus lateralis Gyllenhal, 1833

Brachycerus callosus Schoenherr, 1833

Brachycerus plicatus Gyllenhal, 1833

Brachycerus pradieri Fairmaire, 1856

\section{Curculionidae \\ Bagoinae \\ Bagoini}

Bagous argillaceus Gyllenhal, 1836

Bagous exilis Jacquelin du Val, 1854

Bagous lutosus (Gyllenhal, 1813)

$$
\begin{gathered}
\text { Baridinae } \\
\text { Baridini } \\
\text { Baridina }
\end{gathered}
$$

Arganthonius albomaculatus (H. Brisout, 1867)

Aulacobaris caerulescens (Scopoli, 1763)

Aulacobaris cuprirostris (Fabricius, 1787)

Aulacobaris fallax (H. Brisout, 1870)

Aulacobaris cf. gudenusi (Schultze, 1901)

Aulacobaris ignifer (Fuente, 1901)

Aulacobaris prasina prasina (Boheman, 1836)

Eremobaris picturata opiparis (Jacquelin du Val, 1852)

Labiaticola teruelensis (Hustache, 1928)

Malvaevora timida (Rossi, 1792)

Melaleucus sellatus (Boheman, 1844)

Melanobaris laticollis (Marsham, 1802)

Melanobaris morio (Boheman, 1844)

Melanobaris quadraticollis (Boheman, 1836)

Melanobaris sinapis (Normand, 1937)

$$
\begin{aligned}
& \text { Ceutorhynchinae } \\
& \text { Ceutorhynchini }
\end{aligned}
$$

Ceutorhynchus aeneicollis Germar, 1824

Ceutorhynchus alternans Ch. Brisout, 1869

Ceutorhynchus assimilis (Paykull, 1800)

Ceutorhynchus chalybaeus Germar, 1824

Ceutorhynchus erysimi (Fabricius, 1787)

Ceutorhynchus gallorhenanus Solari, 1949

Ceutorhynchus gracilis Dieckmann \& Smreczyóski, 1972

Ceutorhynchus hirtulus Germar, 1824

Ceutorhynchus insidiosus Ch. Brisout, 1869

E+

Ceutorhynchus leucorhamma Rosenhauer, 1856

Ceutorhynchus minutus (Reich, 1797)

Ceutorhynchus napi Gyllenhal, 1837 
Ceutorhynchus obstrictus (Marsham, 1802)

Ceutorhynchus pallidactylus (Marsham, 1802)

Ceutorhynchus parvulus Ch. Brisout, 1869

Ceutorhynchus perrisi Ch. Brisout, 1869

Ceutorhynchus picitarsis Gyllenhal, 1837

Ceutorhynchus pyrrhorhynchus (Marsham, 1802)

Ceutorhynchus rhenanus (Schultze, 1895)

Ceutorhynchus sardeanensis Schultze, 1903

Ceutorhynchus sophiae Gyllenhal, 1837

Ceutorhynchus sp.

Ceutorhynchus squamulosus Ch. Brisout, 1869

Ceutorhynchus striatellus Schultze, 1900

Ceutorhynchus subpilosus Ch. Brisout, 1869

Ceutorhynchus sulcicollis (Paykull, 1800)

Ceutorhynchus tibialis Boheman, 1845

Ceutorhynchus unguicularis Thomson, 1871

Coeliastes lamii (Fabricius, 1793)

Coeliodes ilicis (Bedel, 1885)

Coeliodes rana (Fabricius, 1787)

Coeliodes ruber (Marsham, 1802)

Coeliodes transversealbofasciatus (Goeze, 1777)

Glocianus distinctus (Ch. Brisout, 1870)

Glocianus lethierryi (Ch. Brisout, 1866)

Glocianus nebulosus (Ch. Brisout, 1866)

Hadroplontus trimaculatus (Fabricius, 1775)

Microplontus rugulosus (Herbst, 1795)

Mogulones abbreviatulus (Fabricius, 1793)

Mogulones arcasi (Ch. Brisout, 1869)

Mogulones geographicus (Goeze, 1777)

Mogulones larvatus (Schultze, 1897)

Mogulones t-album (Gyllenhal, 1837)

Nedyus quadrimaculatus (Linnaeus, 1758)

Neoglocianus maculaalba (Herbst, 1795)

Oprohinus suturalis (Fabricius, 1775)

Parethelcus pollinarius (Forster, 1771)

Prisistus biscutellatus (Chevrolat, 1859)

Ranunculiphilus (Ranunculiphilus) obscurus (Ch. Brisout, 1869)

Stenocarus ruficornis (Stephens, 1831)

Thamiocolus pubicollis (Gyllenhal, 1837)

Thamiocolus subulatus (Ch. Brisout, 1869)

Trichosirocalus barnevillei (Grenier, 1866)

Trichosirocalus briesei Alonso-Zarazaga \& Sánchez-Ruiz, 2002

Trichosirocalus histrix (Perris, 1852)

Trichosirocalus troglodytes (Fabricius, 1787)

Zacladus exiguus (Olivier, 1807)

\section{HYPURINI}

Aphytobius fuentei (Desbrochers, 1908)

Aphytobius sphaerion (Boheman, 1845) [?]

Hypurus bertrandi (Perris, 1852)

Mononychini

Mononychus punctumalbum (Herbst, 1784)

Phytobiini

Neophytobius granatus (Gyllenhal, 1836)

Pelenomus quadrituberculatus (Fabricius, 1787)

$\mathbf{E}+$
Rhinoncus castor (Fabricius, 1793)

Rhinoncus pericarpius (Linnaeus, 1758)

Conoderinae

Coryssomerini

Coryssomerus capucinus (Beck, 1817)

\section{Cossoninae \\ Cossonini}

E Cossonus (Cossonus) linearis (Fabricius, 1775)

Mesites (Mesites) aquitanus Fairmaire, 1859

$$
\text { Onycholipini }
$$

Brachytemnus porcatus (Germar, 1824)

Hexarthrum exiguum (Boheman, 1838)

Stenoscelis (Stenoscelis) submuricatus (Boheman, 1837)

$$
\begin{aligned}
& \text { Rhyncolini } \\
& \text { Phloeophagina }
\end{aligned}
$$

Melicius cylindrus (Boheman, 1838)

Melicius gracilis (Rosenhauer, 1856)

Rhyncolina

Rhyncolus (Axenomimetes) reflexus Boheman, 1838

Rhyncolus (Rhyncolus) ater (Linnaeus, 1758)

Rhyncolus (Rhyncolus) elongatus (Gyllenhal, 1827)

$$
\begin{aligned}
& \text { Cryptorhynchinae } \\
& \text { Cryptorhynchini } \\
& \text { Cryptorhynchina }
\end{aligned}
$$

Cryptorhynchus (Cryptorhynchus) lapathi (Linnaeus, 1758)

Tylodina

Echinodera sp.

$$
\begin{aligned}
& \text { Curculioninae } \\
& \text { Acentrusini }
\end{aligned}
$$

Acentrus histrio (Schoenherr, 1837)

\section{Anthonomini}

Anthonomus (Anthomorphus) phyllocola (Herbst, 1795)

Anthonomus (Anthonomus) rubi (Herbst, 1795)

Anthonomus (Anthonomus) ulmi (DeGeer, 1775)

Anthonomus (Furcipus) rectirostris (Linnaeus, 1758)

Brachonyx pineti (Paykull, 1793)

Bradybatus (Nothops) elongatulus (Boheman, 1843)

Cionini

Cionus alauda (Herbst, 1784)

Cionus hortulanus (Geoffroy, 1785)

Cionus longicollis $\mathrm{Ch}$. Brisout, 1863 
Cionus olivieri Rosenschoeld, 1838

Cionus scrophulariae (Linnaeus, 1758)

Cionus thapsus (Fabricius, 1793)

Stereonychus fraxini (DeGeer, 1775)

$$
\begin{aligned}
& \text { Curculionini } \\
& \text { Curculionina }
\end{aligned}
$$

Archarius ochreatus (Fåhraeus, 1843)

Archarius pyrrhoceras (Marsham, 1802)

Curculio elephas (Gyllenhal, 1836)

Curculio glandium Marsham, 1802

Curculio pellitus (Boheman, 1843)

Curculio venosus (Gravenhorst, 1807)

Curculio villosus Fabricius, 1781

$$
\begin{gathered}
\text { Ellescini } \\
\text { Dorytomina }
\end{gathered}
$$

Dorytomus (Dorytomus) dejeani Faust, 1883

Dorytomus (Dorytomus) edoughensis Desbrochers, 1875

Dorytomus (Dorytomus) longimanus (Forster, 1771)

Dorytomus (Olamus) melanophthalmus (Paykull, 1793)

\section{Mecinini}

Cleopomiarus distinctus (Boheman, 1845)

Cleopomiarus meridionalis (H. Brisout, 1862)

Cleopomiarus plantarum (Germar, 1824)

Gymnetron beccabungae (Linnaeus, 1761)

Gymnetron rostellum (Herbst, 1795)

Gymnetron villosulum Gyllenhal, 1838

Mecinus circulatus (Marsham, 1802)

Mecinus sp.

Mecinus pascuorum (Gyllenhal, 1813)

Rhinusa antirrhini (Paykull,1800)

Rhinusa tetra (Fabricius, 1793)

Rhinusa vestita (Germar, 1821)

\section{Rhamphini \\ Rhamphina}

Isochnus foliorum (Müller, 1776)

Orchestes (Orchestes) avellanae (Donovan, 1797)

Orchestes (Orchestes) erythropus tricolor Kiesenwetter, 1851

Orchestes (Orchestes) irroratus Kiesenwetter, 1852

Orchestes (Orchestes) pilosus (Fabricius, 1781)

Orchestes (Orchestes) quercus (Linnaeus, 1758)

Rhamphus (Rhamphus) pulicarius (Herbst, 1795)

Rhamphus (Rhamphus) subaeneus (Illiger, 1807)

$$
\text { Smicronychini }
$$

Smicronyx (Smicronyx) jungermanniae (Reich, 1797) Smicronyx (Smicronyx) reichi (Gyllenhal, 1836)

\section{Storeini}

Pachytychius asperatus (Dufour, 1843)

Pachytychius haematocephalus (Gyllenhal, 1835)

Pachytychius hordei squamosus (Gyllenhal, 1836)
Pachytychius maculosus Reitter, 1907

Pachytychius sparsutus (Olivier, 1807)

$$
\text { Styphlini }
$$

Orthochaetes extensus (Chevrolat, 1880)

Orthochaetes rectirostris Hoffmann, 1960

Orthochaetes setiger (Beck, 1817)

Tychiini

Lignyodina

Lignyodes enucleator (Panzer, 1798)

Tychiina

Sibinia (Dichotychius) sodalis Germar, 1824

Sibinia (Sibinia) arenariae Stephens, 1831

Sibinia (Sibinia) attalica Gyllenhal, 1835

Sibinia (Sibinia) femoralis Germar, 1824

Sibinia (Sibinia) iberica Hoffmann, 1960

Sibinia (Sibinia) pellucens (Scopoli, 1772)

Sibinia (Sibinia) primita (Herbst, 1795)

Sibinia (Sibinia) pyrrhodactyla (Marsham, 1802)

Sibinia (Sibinia) subelliptica Desbrochers, 1873

Sibinia (Sibinia) variata Gyllenhal, 1835

Tychius (Tychius) argentatus Chevrolat, 1859

Tychius (Tychius) aurichalceus Gyllenhal, 1836

Tychius (Tychius) cinnamomeus Kiesenwetter, 1852

Tychius (Tychius) consputus Kiesenwetter, 1851

Tychius (Tychius) cuprifer (Panzer, 1799)

Tychius (Tychius) cuprinus Rosenhauer, 1856

Tychius (Tychius) cylindricollis Solari, 1950

Tychius (Tychius) elegans Desbrochers, 1896

Tychius (Tychius) elegantulus Ch. Brisout, 1862

Tychius (Tychius) farinosus Rosenhauer, 1856

Tychius (Tychius) funicularis Ch. Brisout, 1862

Tychius (Tychius) fuscipes Chevrolat, 1859

Tychius (Tychius) grenieri Ch. Brisout, 1861

Tychius (Tychius) hispanus Velázquez de Castro \& Caldara, 1992

Tychius (Tychius) laticollis Perris, 1864

Tychius (Tychius) longicollis Ch. Brisout, 1862

Tychius (Tychius) parallelus (Panzer, 1794)

Tychius (Tychius) picirostris (Fabricius, 1787)

Tychius (Tychius) polylineatus (Germar, 1824)

Tychius (Tychius) pusillus Germar, 1824

Tychius (Tychius) quinquepunctatus (Linnaeus, 1758)

Tychius (Tychius) rufipennis Ch. Brisout, 1862

Tychius (Tychius) squamulatus Gyllenhal, 1836

Tychius (Tychius) tibialis Boheman, 1843

Cyclominae

Dichotrachelini

Dichotrachelus dellabeffai Meregalli, 1982 [?]

Dichotrachelus graellsii graellsii Perris, 1864

$$
\begin{gathered}
\text { Rhythirrinini } \\
\text { Gronopina }
\end{gathered}
$$

Entomoderus (Pseudorhinus) bonvouloirii (Ch. Brisout, 1866) 
Entomoderus (Pseudorhinus) caudatus (Ch. Brisout, 1866)

Entomoderus (Pseudorhinus) interruptus (Ch. Brisout, 1866)

Entomoderus (Pseudorhinus) laesirostris stenoderus (Gemminger, 1871)

Entomoderus (Pseudorhinus) longulus longulus (Rosenhauer, 1856)

Entomoderus (Pseudorhinus) oberthueri (Desbrochers, 1910)

Entomoderus (Pseudorhinus) tenuipes (Solari, 1940) [?]

Entomoderus (Pseudorhinus) variabilis (Ch. Brisout, 1866)

Gronops luctuosus Chevrolat, 1861

Gronops lunatus lunatus (Fabricius, 1775)

Entiminae

Alophini

Rhytideres plicatus (Olivier, 1790)

Brachyderini

Brachyderes (Brachyderes) grisescens Fairmaire, 1862 Brachyderes (Brachyderes) incanus (Linnaeus, 1758)

Brachyderes (Brachyderes) lineolatus Fairmaire, 1862 Brachyderes (Brachyderes) pubescens Boheman, 1833 Brachyderes (Brachyderes) suturalis Graells, 1851 Brachyderes (Brachylophus) lusitanicus (Fabricius, 1781) Brachyderes (Gastraspis) marginellus Graells, 1858 E Strophomorphus porcellus (Schoenherr, 1832) Strophosoma (Neliocarus) ebenista (Seidlitz, 1870) Strophosoma (Neliocarus) faber (Herbst, 1784) Strophosoma (Neliocarus) laufferi (Flach, 1907) Strophosoma (Strophosoma) erinaceus (Chevrolat, 1865) Strophosoma (Strophosoma) melanogrammum melanogrammum (Forster, 1771)

\section{Cneorhinini}

Attactagenus carinirostris (Boheman, 1840)

Attactagenus ceballosi Viedma, 1965

Attactagenus cordubensis (Kirsch, 1870)

Attactagenus diecki (Tournier, 1874)

Attactagenus dispar (Graells, 1858)

Attactagenus moralesi Viedma, 1965

Attactagenus pyriformis (Boheman, 1833)

Attactagenus spinipes (Pérez Arcas, 1872)

Attactagenus tarsalis (Tournier, 1874)

Cneorhinus argentifer Marseul, 1866

Philopedon plagiatum (Schaller, 1783)

Polydius hispanus hispanus (Herbst, 1797)

\section{Otiorhynchini}

Otiorhynchus (Arammichnus) cribricollis Gyllenhal, 1834

Otiorhynchus (Dorymerus) sulcatus (Fabricius, 1775)

Otiorhynchus (Harpinorrhynchus) dentipes dentipes

Graells, 1858
Otiorhynchus (Jelenantus) affaber Boheman, 1843

Otiorhynchus (Metopiorrhynchus) veterator Uyttenboogaart, 1932

Otiorhynchus (Otiorhynchus) truncatellus Graells, 1858

Otiorhynchus (Petalorrhynchus) validus Stierlin, 1881

$\mathbf{E}$

Otiorhynchus (Zustalestus) rugosostriatus (Goeze, 1777)

Peritelini

Pseudomeira prolixa (Kiesenwetter, 1851)

Phyllobiini

Phyllobius (Phyllobius) pyri (Linnaeus, 1758)

Phyllobius (Phyllobius) squamosus Ch. Brisout, 1866

Phyllobius (Phyllobius) tuberculifer Chevrolat, 1865

Phyllobius (Subphyllobius) virideaeris virideaeris charting, 1781)

Polydrusini

Homapterus punctulatus (Ch. Brisout, 1866)

Pachyrhinus glabratus (Chevrolat, 1866)

Pachyrhinus squamosus (Kiesenwetter, 1852)

Polydrusus (Chaerodrys) setifrons Jacquelin du Val, 1852

Polydrusus (Chomatocryptus) xanthopus Gyllenhal, 1834

Polydrusus (Eurodrusus) chrysomela (Olivier, 1807) E

Polydrusus (Eurodrusus) confluens Stephens, 1831

Polydrusus (Eustolus) corruscus Germar, 1824

Polydrusus (Eustolus) impressifrons Gyllenhal, 1834

Polydrusus (Eustolus) interstitialis Perris, 1864

Polydrusus (Eustolus) pterygomalis Boheman, 1840

Polydrusus (Leucodrusus) senex Chevrolat, 1866

Polydrusus (Leucodrusus) tibialis Gyllenhal, 1834

Polydrusus (Neoeustolus) pilosulus Chevrolat, 1865 
Sitona (Sitona) puberulus Reitter, 1903

Sitona (Sitona) puncticollis Stephens, 1831

Sitona (Sitona) regensteinensis (Herbst, 1797)

Sitona (Sitona) sulcifrons argutulus Gyllenhal, 1834

Sitona (Sitona) tenuis Rosenhauer, 1847

\section{Tanymecini}

Tanymecina

Cycloderes (Cycloderes) argenteus (Perris, 1866)

Cycloderes (Cycloderes) glabratus (Gyllenhal, 1833)

Cycloderes (Cycloderes) heliophilus (Chevrolat, 1866)

Cycloderes (Cycloderes) latithorax (Desbrochers, 1872) E

Cycloderes (Cycloderes) tessellatus (Gyllenhal, 1833) $\mathbf{E}$

Cycloderes (Cycloderes) tonsus (Chevrolat, 1866)

Trachyphloeini

Trachyphloeina

Cathormiocerus (Cathormiocerus) carpetanus Uhagón, 1885

Cathormiocerus (Cathormiocerus) chevrolati Seidlitz, 1868

Cathormiocerus (Cathormiocerus) horrens horrens (Gyllenhal, 1834)

Cathormiocerus (Cathormiocerus) myrmecophilus

(Seidlitz, 1868)

Cathormiocerus (Cathormiocerus) porculus Uhagón, 1885

Cathormiocerus (Cathormiocerus) socius Boheman, 1843

Cathormiocerus (Mitomermus) brevirostris (Ch. Brisout, 1866)

Cathormiocerus (Schaumius) excursor Stierlin, 1868

Cathormiocerus (Schaumius) irrasus irrasus Seidlitz, 1868

E

Cathormiocerus (Schaumius) lapidicola Chevrolat, 1866 E

Cathormiocerus (Schaumius) lethierryi Chevrolat, 1866

Cathormiocerus (Schaumius) pygmaeus Seidlitz, 1868

Cathormiocerus sp.

Trachyphloeus (Lacordairius) seidlitzii (Ch. Brisout, 1866)

Trachyphloeus (Trachyphloeus) angustisetulus Hansen, 1915

Trachyphloeus (Trachyphloeus) guadarramus Seidlitz, 1868

Hyperinae
Hyperini

Coniatus (Bagoides) suavis Gyllenhal, 1834

Coniatus (Coniatus) repandus (Fabricius, 1793)

Donus (Antidonus) brevirostris (Capiomont, 1868) $\quad$ E+

Donus (Antidonus) dauci (Olivier, 1807)

Donus (Antidonus) lunatus (Wollaston, 1854)

Donus (Antidonus) zoilus (Scopoli, 1763)

Donus (Donus) hispanicus (Capiomont, 1868)

Donus (Donus) cf. hispanicus (Capiomont, 1868)

Donus (Donus) perrisi (Capiomont, 1868)

Donus (Donus) cf. perrisi (Capiomont, 1868)
Hypera (Dapalinus) striata (Boheman, 1834)

Hypera (Hypera) constans (Boheman, 1834)

Hypera (Hypera) fuscocinerea (Marsham, 1802)

Hypera (Hypera) lethierryi (Capiomont, 1868)

Hypera (Hypera) nigrirostris (Fabricius, 1775)

Hypera (Hypera) plantaginis (DeGeer, 1775)

Hypera (Hypera) postica (Gyllenhal, 1813)

Hypera (Hypera) venusta (Fabricius, 1781)

Hypera (Kippenbergia) arator (Linnaeus, 1758)

Hypera (Tigrinellus) pastinacae (Rossi, 1790)

Limobius borealis borealis (Paykull, 1792)

Limobius mixtus Boheman, 1834

\section{Lixinae}

Cleonini

Bothynoderes affinis (Schrank, 1781)

Cleonis pigra (Scopoli, 1763)

Coniocleonus cicatricosus (Hoppe,1795)

Coniocleonus excoriatus (Gyllenhal, 1834)

Coniocleonus graellsii Chevrolat, 1873

Coniocleonus nigrosuturatus (Goeze, 1777)

Coniocleonus sejunctus (Faust, 1904)

Cyphocleonus hedenborgi (Fåhraeus, 1842)

Ephimeronotus miegii (Fairmaire,1855)

Leucomigus tesselatus (Fairmaire, 1849)

Mecaspis alternans (Herbst, 1795)

Pseudocleonus (Pseudocleonus) carinatus (Gyllenhal, 1834)

Xanthochelus cinctiventris marmottani (Ch. Brisout, 1866)

\section{Lixini}

Larinus (Cryphopus) cf. bombycinus Lucas, 1846

Larinus (Larinomesius) meridionalis Rosenhauer, 1856

Larinus (Larinomesius) of. meridionalis Rosenhat 1856

Larinus (Larinomesius) obtusus australis Capiomont, 1874

Larinus (Larinomesius) scolymi (Olivier, 1807)

Larinus (Larinus) cynarae (Fabricius, 1787)

Larinus (Larinus) flavescens Germar, 1824

Larinus (Larinus) ursus (Fabricius, 1793) s.l.

Larinus (Phyllonomeus) albocinctus Chevrolat, 1866

Larinus (Phyllonomeus) escorialensis Ch. Brisout, 1866

Larinus (Phyllonomeus) jaceae (Fabricius, 1775) [?]

Larinus (Phyllonomeus) lethierryi Ch. Brisout, 1866 E

Larinus (Phyllonomeus) longirostris Gyllenhal, 1835

Larinus (Phyllonomeus) planus (Fabricius, 1793)

Larinus (Phyllonomeus) sturnus (Schaller, 1783)

Larinus (Phyllonomeus) turbinatus Gyllenhal, 1835

Lixus (Compsolixus) albomarginatus Boheman, 1843

Lixus (Compsolixus) anguinus (Linnaeus, 1767)

E Lixus (Compsolixus) ascanii (Linnaeus, 1767) [?]

E Lixus (Compsolixus) castellanus Chevrolat, 1865

Lixus (Compsolixus) laufferi Petri, 1905 
Lixus (Dilixellus) pulverulentus (Scopoli,1763)

Lixus (Dilixellus) punctiventris Boheman, 1835

Lixus (Dilixellus) rumicis Hoffmann, 1956

Lixus (Dilixellus) vilis (Rossi, 1790)

Lixus (Epimeces) cardui Olivier, 1807

Lixus (Epimeces) filiformis (Fabricius, 1781)

Lixus (Epimeces) flaveolus Motschulsky, 1849

Lixus (Epimeces) scolopax Boheman, 1835

Lixus (Epimeces) ulcerosus Petri, 1904

Lixus (Eulixus) brevipes brevipes Ch. Brisout, 1866

Lixus (Eulixus) brevirostris Boheman, 1836

Lixus (Eulixus) iridis Olivier, 1807

Lixus (Eulixus) scabricollis Boheman, 1843

Lixus (Lixoglyptus) spartii Olivier, 1807

Lixus (Ortholixus) acicularis Germar, 1824

Lixus (Ortholixus) elegantulus Boheman, 1843

Lixus (Ortholixus) mucronatus (Olivier, 1790)

Lixus (Ortholixus) puncticollis Ch. Brisout, 1866

Microlarinus lareynii (Jacquelin du Val, 1852)

Rhinocyllini

Rhinocyllus conicus (Froelich, 1792)

\section{Mesoptiliinae \\ Magdalidini}

Magdalis (Laemosaccidius) exarata Ch. Brisout, 1862 Magdalis (Magdalis) duplicata Germar, 1824 Magdalis (Magdalis) frontalis (Gyllenhal, 1827)

Magdalis (Magdalis) memnonia (Gyllenhal, 1827)

Magdalis (Magdalis) phlegmatica (Herbst, 1797)

Magdalis (Magdalis) rufa (Germar, 1824)

Magdalis (Magdalis) violacea (Linnaeus, 1758)

Magdalis (Odontomagdalis) armigera (Geoffroy, 1785)

Magdalis (Panus) barbicornis (Latreille, 1804)

$$
\begin{aligned}
& \text { Molytinae } \\
& \text { Hylobiini } \\
& \text { Hylobiina }
\end{aligned}
$$

Hylobius (Callirus) abietis (Linnaeus, 1758)

$$
\begin{aligned}
& \text { Molytini } \\
& \text { Molytina }
\end{aligned}
$$

Anisorhynchus hespericus Desbrochers, 1875

$$
\text { Plinthina }
$$

Iberoplinthus perezii (Ch. Brisout, 1866)

$$
\begin{aligned}
& \text { Pissodini } \\
& \text { Pissodina }
\end{aligned}
$$

Pissodes (Pissodes) castaneus (DeGeer, 1775)

Pissodes (Pissodes) pini (Linnaeus, 1758)

Pissodes (Pissodes) piniphilus (Herbst, 1797)

Pissodes (Pissodes) validirostris (C.R. Sahlberg, 1834)
Scolytinae

Corthylini

E Pityophthorus glabratus Eichhoff, 1871

$$
\text { Crypturgini }
$$

E Crypturgus cinereus (Herbst, 1793)

Crypturgus cribrellus (Reitter, 1894)

Crypturgus mediterraneus Eichhoff, 1869

Crypturgus numidicus Ferrari, 1867

$$
\text { Hylastini }
$$

Hylastes angustatus (Herbst, 1793)

Hylastes ater (Paykull, 1800)

Hylastes attenuatus Erichson, 1836

Hylastes linearis Erichson, 1836

Hylurgops glabratus (Zetterstedt, 1828)

E Hylurgops palliatus (Gyllenhal, 1813)

\section{Hylesinini}

Hylesinus crenatus (Fabricius, 1787)

Hylesinus varius (Fabricius, 1775)

Pteleobius kraatzi (Eichhoff, 1864)

Pteleobius vittatus (Fabricius, 1793)

Hypoborini

Hypoborus ficus Erichson, 1836

$$
\text { Ipini }
$$

Ips (Cumatotomicus) sexdentatus (Boerner, 1767) Ips (Cyrtotomicus) acuminatus (Gyllenhal, 1827) Orthotomicus erosus (Wollaston, 1857)

Orthotomicus laricis (Fabricius, 1793)

Orthotomicus longicollis (Gyllenhal, 1827)

Orthotomicus proximus (Eichhoff, 1868)

Pityogenes bidentatus (Herbst, 1784)

Pityogenes calcaratus (Eichhoff,1878)

Pityogenes quadridens (Hartig, 1834)

$$
\text { Phloeotribini }
$$

Phloeotribus rhododactylus (Marsham, 1802)

Phloeotribus scarabaeoides (Bernard, 1788)

\section{Scolytini}

E Scolytus carpini (Ratzeburg, 1837)

Scolytus kirschii fasciatus Reitter, 1890

Scolytus multistriatus (Marsham, 1802)

Scolytus pygmaeus (Fabricius, 1787)

E Scolytus rugulosus (Müller, 1818)

Scolytus scolytus (Fabricius, 1775)

Tomicini

Hylurgus ligniperda (Fabricius, 1787)

Hylurgus micklitzi Wachtl, 1881

Tomicus minor (Hartig, 1834)

Tomicus piniperda (Linnaeus, 1758) 
Xyleborini

Xyleborinus attenuatus (Blandford, 1894)

Xyleborinus saxesenii (Ratzeburg, 1837)

Xyleborus eurygraphus (Ratzeburg, 1837)

Xyloterini

Trypodendron lineatum (Olivier, 1795)

Trypodendron signatum (Fabricius, 1793)

\section{DRYOPHTHORIDAE \\ Rhynchophorinae Litosomini}

Sitophilus zeamais Motschulsky, 1855

Sphenophorini

Sphenophorus parumpunctatus Gyllenhal, 1838

Sphenophorus piceus (Pallas, 1776)

Sphenophorus striatopunctatus (Goeze, 1777)

\section{ERIRHINIDAE \\ Erirhininae \\ Erirhinini}

Notaris scirpi (Fabricius, 1793)

\section{NANOPHYIDAE \\ Nanophyinae \\ Corimaliini}

Corimalia postica (Gyllenhal, 1838)

Corimalia tamarisci (Gyllenhal, 1838)

Corimalia tetrastigma (Aubé, 1863)

Hypophyes minutissimus (Tournier, 1867)

Hypophyes pallidulus (Gravenhorst, 1807)

\section{Nanophyini}

Ctenomeropsis nigra (Waltl, 1835)

Dieckmanniellus gracilis (Redtenbacher, 1849)

Dieckmanniellus nitidulus (Gyllenhal, 1838)

Nanomimus hemisphaericus (Olivier, 1807)

Nanophyes brevis bleusei Pic, 1900

Nanophyes rubricus Rosenhauer, 1856

Pericartiellus flavidus (Aubé, 1850)

\section{NEMONYCHIDAE \\ Cimberidinae \\ Doydirhynchini}

Doydirhynchus austriacus (Olivier, 1807)

\author{
RHYNCHITIDAE \\ Rhynchitinae \\ Auletini \\ Auletina
}

Auletes tubicen Schoenherr, 1826

Auletobius (Mesauletobius) pubescens (Kiesenwetter, 1852)
Rhynchitini

Rhynchitina

Haplorhynchites (Haplorhynchites) pubescens (Fabricius, 1775)

Lasiorhynchites (Coccygorrhynchites) sericeus (Herbst, 1797)

Lasiorhynchites (Stenorhynchites) caeruleocephalus (Schaller, 1783)

Neocoenorrhinus interpunctatus (Stephens, 1831)

Rhynchites (Epirhynchites) auratus (Scopoli, 1763)

Rhynchites (Rhynchites) bacchus (Linnaeus, 1758)

Tatianaerhynchites aequatus (Linnaeus, 1767)

Temnocerus tomentosus (Gyllenhal, 1839)

\section{Conclusión}

Los táxones de Curculionoidea de la CM siguen el patrón usual de la diversidad de este grupo en la Península; sin embargo, con los datos de que disponemos en este momento podemos asegurar que el número de táxones nuevos para la $\mathrm{CM}$ deberá aumentar con las prospecciones adecuadas. Se ofrece como colofón una tabla con los datos numéricos por familias:

$\begin{array}{lccc}\text { Familia } & \begin{array}{c}\mathbf{N}^{\mathbf{0}} \mathbf{d e} \\ \text { géneros }\end{array} & \begin{array}{c}\mathbf{N}^{\mathbf{0}} \mathbf{d e} \\ \text { especies }\end{array} & \begin{array}{r}\text { Especies } \\ \text { dudosas }\end{array} \\ \text { Anthribidae } & 4 & 5 & \\ \text { Apionidae } & 26 & 84 & 5 \\ \text { Attelabidae } & 1 & 1 & \\ \text { Brachyceridae } & 1 & 4 & 4 \\ \text { Curculionidae } & 124 & 430 & \\ \text { Dryopthoridae } & 2 & 4 & \\ \text { Erirhinidae } & 1 & 1 & \\ \text { Nanophyidae } & 7 & 12 & \\ \text { Nemonychidae } & 1 & 1 & \\ \text { Rhynchitidae } & 8 & 10 & \\ \text { Total: } & \mathbf{1 7 5} & \mathbf{5 5 2} & \end{array}$

Al comparar estos datos con los presentados por Alonso-Zarazaga (2002) para todo el área iberobalear, queda patente que la CM es una de las zonas mejor conocidas, habiéndose encontrado en ella el $71,4 \%$ de las familias, el $46,9 \%$ de los géneros y el $32 \%$ de las especies halladas en la Península Ibérica e Islas Baleares hasta el momento, cifras a las que sólo se acerca Cataluña.

\section{Referencias}

Alonso-Zarazaga, M. A., 2002. Lista preliminar de los Coleoptera Curculionoidea del área ibero-balear, con descripción de Melicius gen. nov. y nuevas citas. Boletín de la Sociedad Entomológica Aragonesa, 31: 9-33. 
Alonso-Zarazaga, M. A. \& Lyal, C. H. C., 1999. A World Catalogue of Families and Genera of Curculionoidea (Insecta: Coleoptera) (Excepting Scolytidae and Platypodidae). Entomopraxis. Barcelona. 315 pp.

Alonso-Zarazaga, M. A. \& Lyal, C. H. C., 2002. Addenda and corrigenda to 'A World Catalogue of Families and Genera of Curculionoidea (Insecta: Coleoptera)'. Zootaxa, 63: 1-37.
EscalerA, M. M. DE LA, 1919. Ipidos (Scolytidos) observados en la Península Ibérica, Marruecos y Canarias. Boletín de la Real Sociedad Española de Historia Natural, 19(2): 103-108.

Iglesias Iglesias, L., 1922. Enumeración de los Curculiónidos de la Península Ibérica e Islas Baleares. Boletín de la Real Academia de Ciencias Exactas, Físicas y Naturales, 18: 1-117. 